\title{
PORTFOLIO OPTIMIZATION WITH UNOBSERVABLE MARKOV-MODULATED DRIFT PROCESS
}

\author{
ULRICH RIEDER, ${ }^{*}$ University of Ulm \\ NICOLE BÄUERLE, ${ }^{* *}$ University of Hannover
}

\begin{abstract}
We study portfolio optimization problems in which the drift rate of the stock is Markov modulated and the driving factors cannot be observed by the investor. Using results from filter theory, we reduce this problem to one with complete observation. In the cases of logarithmic and power utility, we solve the problem explicitly with the help of stochastic control methods. It turns out that the value function is a classical solution of the corresponding Hamilton-Jacobi-Bellman equation. As a special case, we investigate the so-called Bayesian case, i.e. where the drift rate is unknown but does not change over time. In this case, we prove a number of interesting properties of the optimal portfolio strategy. In particular, using the likelihood-ratio ordering, we can compare the optimal investment in the case of observable drift rate to that in the case of unobservable drift rate. Thus, we also obtain the sign of the drift risk.
\end{abstract}

Keywords: Portfolio optimization; Markov-modulated drift; Hamilton-Jacobi-Bellman equation; optimal investment strategy; Bayesian control; stochastic ordering

2000 Mathematics Subject Classification: Primary 93E20

\section{Introduction}

In this paper, we investigate the problem of optimal portfolio choice in a financial market with one bond and one stock. The drift rate of the stock price process is modelled as a continuous-time Markov chain. The aim is to maximize the expected utility from terminal wealth. However, we are only able to observe the stock price process and have to base our decision on this observation. In particular, we are not informed about the state of the drift process. Such a model is also called a hidden Markov model. For a general treatment of such models see, e.g. Elliott et al. (1994).

It is well known that a financial market with constant parameters can only serve for a relatively short period of time. Thus, it is necessary to use stochastically varying parameters. One possibility is to introduce a continuous-time Markov chain, representing the general market direction. For simplicity, we assume that only the drift rate of the stock price process depends on this market direction. Furthermore, it seems to be realistic that we cannot directly observe this market direction since not all the driving factors, or their impacts, are known. Thus, we can only try to estimate this hidden factor by observing the stock price.

Portfolio optimization problems with partial observation, in particular with unknown drift process, have been studied extensively over the last decade. Lakner (1995), (1998) and

Received 12 October 2004; revision received 25 November 2004.

* Postal address: Department of Optimization and Operations Research, University of Ulm, D-89069 Ulm, Germany. Email address: rieder@mathematik.uni-ulm.de

** Postal address: Institute for Mathematical Stochastics, University of Hannover, D-30167 Hannover, Germany. Email address: baeuerle@stochastik.uni-hannover.de 
Rishel (1999), for example, have treated the case in which the drift rate follows a linear Gaussian model. Karatzas and Zhao (2001) investigated the Bayesian case, i.e. where the drift rate is constant but unknown. The papers by Sass and Haussmann (2004) and Haussmann and Sass (2004) discuss a market model that is even more general than ours, for example in allowing a stochastic interest rate and assuming $d$ risky assets. Since it is possible to reduce the problem to one in a complete financial market, these cited papers use the martingale approach to solve the portfolio problem. The only exceptions are Rishel (1999) and Karatzas and Zhao (2001), who use a stochastic control approach. Portfolio optimization problems with observable Markovmodulated market data have been treated in Bäuerle and Rieder (2004).

In this paper, we use a stochastic control approach for the portfolio optimization problem with unobservable Markov-modulated drift process. The first contribution of our paper is that we can show that this approach works very well in the cases of logarithmic and power utility, in the sense that we even obtain a classical solution of the corresponding Hamilton-Jacobi-Bellman (HJB) equation. This is of practical interest since it gives an alternative way of computing the value function and the optimal portfolio strategy. As a special case, we also investigate the so-called Bayesian case, i.e. where the drift rate is unknown but does not change over time. This setting has already been investigated in Karatzas and Zhao (2001); however, we give a self-contained approach to the problem, treating it as a special case of the hidden Markovmodulated drift model, and derive a more explicit formula for the optimal investment strategy. We prove a number of interesting properties of the optimal portfolio strategy, in particular, when compared to the case of observable drift rate. For example, when we have a power utility $u(x)=x^{\alpha} / \alpha$, with $\alpha \in(0,1)$, it turns out that we have to invest more in the stock in the case of an unobservable drift rate, relative to the case in which the drift rate is known and equal to our expectation. If $\alpha<0$, this conclusion is reversed. Thus, for $\alpha \in(0,1)$, the drift risk is positive whereas, for $\alpha<0$, the drift risk is negative. This result is obtained by using the likelihood-ratio ordering in an appropriate way. Some numerical results are also presented.

The paper is organized as follows. In Section 2, we introduce the market model and define the optimization problem. In Section 3, we use filtering theory to reduce the problem to one with complete observation. In the case of a logarithmic utility function, the problem is solved in Section 4. In Section 5, we treat the case of a power utility. With the help of a stochastic control approach, we are able to solve the problem; in particular, it turns out that the value function is a classical solution of the corresponding HJB equation. We show this in Section 6. The special Bayesian case is treated in Section 7 and properties of the optimal investment strategy are proven in Section 8.

\section{The model}

Suppose that $\left(\Omega, \mathcal{F}, \mathfrak{F}=\left\{\mathscr{F}_{t}, 0 \leq t \leq T\right\}, \mathrm{P}\right)$ is a filtered probability space with filtration $\mathfrak{F}=\left\{\mathcal{F}_{t}, 0 \leq t \leq T\right\}$, and that $T>0$ is a fixed time horizon. We consider a financial market with one bond and one risky asset. The bond price process $B=\left(B_{t}\right)$ evolves according to

$$
\mathrm{d} B_{t}=r B_{t} \mathrm{~d} t
$$

with $r>0$ being the interest rate. The stock price process $S=\left(S_{t}\right)$ is given by

$$
\mathrm{d} S_{t}=S_{t}\left(\mu_{t} \mathrm{~d} t+\sigma \mathrm{d} W_{t}\right)
$$

where $\mu_{t}=\boldsymbol{\mu}^{\top} \boldsymbol{Y}_{t}, W=\left(W_{t}\right)$ is a Brownian motion, and $\boldsymbol{Y}=\left(\boldsymbol{Y}_{t}\right)$ is a continuous-time Markov chain with state space $\left\{\boldsymbol{e}_{1}, \ldots, \boldsymbol{e}_{d}\right\}$, where $\boldsymbol{e}_{k}$ is the $k$ th unit vector in $\mathbb{R}^{d}$ and $\left(\boldsymbol{Y}_{t}\right)$ has 
the generator $\boldsymbol{Q}=\left(q_{i j}\right)$. All processes are adapted with respect to $\mathfrak{F}$, and $\left(W_{t}\right)$ and $\left(\boldsymbol{Y}_{t}\right)$ are independent. Finally, $\boldsymbol{\mu}=\left(\mu_{1}, \ldots, \mu_{d}\right) \in \mathbb{R}^{d}$ and $\sigma>0$.

The optimization problem is to find self-financing investment strategies in this market that maximize the expected utility from terminal wealth. We assume that our investor is only able to observe the stock price process and that he knows the initial distribution of $\boldsymbol{Y}_{0}$. In particular, he is not informed about the current state of the Markov chain. Let $\mathfrak{F}^{S}=\left\{\mathcal{F}_{t}^{S}, 0 \leq t \leq T\right\}$ be the filtration generated by the stock price process $\left(S_{t}\right)$. In what follows, we denote by $\pi_{t} \in \mathbb{R}$ the fraction of wealth invested in the stock at time $t$. Then, $1-\pi_{t}$ is the fraction of wealth invested in the bond at time $t$. If $\pi_{t}<0$ then this means that the stock is sold short, while $\pi_{t}>1$ corresponds to a credit. The process $\pi=\left(\pi_{t}\right)$ is called a portfolio strategy. An admissible portfolio strategy has to be an $\mathfrak{F}^{S}$-adapted process.

The wealth process under an admissible portfolio strategy $\pi$ is given by the solution of the stochastic differential equation

$$
\mathrm{d} \tilde{X}_{t}^{\pi}=\tilde{X}_{t}^{\pi}\left[\left(r+\left(\mu_{t}-r\right) \pi_{t}\right) \mathrm{d} t+\sigma \pi_{t} \mathrm{~d} W_{t}\right],
$$

where we assume that $\tilde{X}_{0}^{\pi}=x_{0}$ is the given initial wealth. We denote by

$$
\begin{aligned}
\mathcal{U}[t, T]:=\{\pi= & \left(\pi_{s}\right)_{t \leq s \leq T}: \pi_{s} \in \mathbb{R}, \pi \text { is } \mathfrak{F}^{S} \text {-adapted, } \\
& \text { (1) has a unique solution, and } \left.\int_{t}^{T}\left(\pi_{s} \tilde{X}_{s}^{\pi}\right)^{2} \mathrm{~d} s<\infty \text { almost surely }\right\}
\end{aligned}
$$

the set of admissible portfolio strategies over the time horizon $[t, T]$. Let $U: \mathbb{R}_{+} \rightarrow \mathbb{R}$ be an increasing, concave, and differentiable utility function. The value functions for our problem are defined by

$$
\begin{aligned}
\tilde{V}_{\pi}(t, x) & =\mathrm{E}^{t, x}\left[U\left(\tilde{X}_{T}^{\pi}\right)\right] \quad \text { for all } \pi \in \mathcal{U}[t, T], \\
\tilde{V}(t, x) & =\sup _{\pi \in \mathcal{U}[t, T]} \tilde{V}_{\pi}(t, x),
\end{aligned}
$$

where $\mathrm{E}^{t, x}$ is the conditional expectation given that $\tilde{X}_{t}^{\pi}=x$. A portfolio strategy $\pi^{*} \in \mathcal{U}[0, T]$ is optimal if $\tilde{V}\left(0, x_{0}\right)=\tilde{V}_{\pi^{*}}\left(0, x_{0}\right)$. Note that $\tilde{V}\left(0, x_{0}\right)$ depends on the initial distribution of $\boldsymbol{Y}_{0}$.

\section{The reduction}

We can reduce the control problem with partial observation to one with complete observation as follows. Denote by

$$
p_{k}(t):=\mathrm{P}\left(\boldsymbol{Y}_{t}=\boldsymbol{e}_{k} \mid \mathcal{F}_{t}^{S}\right), \quad k=1, \ldots, d,
$$

the Wonham filter of the Markov chain, and define $\boldsymbol{p}_{t}=\left(p_{1}(t), \ldots, p_{d}(t)\right)$ (cf. Elliott et al. (1994)). The following statements hold.

Lemma 1. There exists a Brownian motion $\left(\hat{W}_{t}\right)$, with respect to $\mathfrak{F}^{S}$, such that

(a) the filter processes $p_{k}(t)$ satisfy

$$
p_{k}(t)=p_{k}(0)+\int_{0}^{t} \sum_{j} q_{j k} p_{j}(s) \mathrm{d} s+\int_{0}^{t} \frac{1}{\sigma}\left(\mu_{k}-\hat{\mu}_{s}\right) p_{k}(s) \mathrm{d} \hat{W}_{s} \quad \text { for } t \geq 0,
$$

where $\hat{\mu}_{t}:=\sum_{k=1}^{d} \mu_{k} p_{k}(t)=\mathrm{E}\left[\mu_{t} \mid \mathcal{F}_{t}^{S}\right]$; 
(b) $\mu_{t} \mathrm{~d} t+\sigma \mathrm{d} W_{t}=\hat{\mu}_{t} \mathrm{~d} t+\sigma \mathrm{d} \hat{W}_{t}$; and

(c) $\mathcal{F}_{t}^{S}=\sigma\left(\hat{W}_{s}, s \leq t\right)$.

Note that part (b) implies that $\hat{W}_{t}:=W_{t}+(1 / \sigma) \int_{0}^{t}\left(\mu_{s}-\hat{\mu}_{s}\right) \mathrm{d} s$. The control model with complete observation is now characterized, for $\pi \in \mathcal{U}[0, T]$, by the following $(d+1)$ dimensional state process:

$$
\begin{aligned}
\mathrm{d} X_{t}^{\pi} & =X_{t}^{\pi}\left[\left(r+\left(\hat{\mu}_{t}-r\right) \pi_{t}\right) \mathrm{d} t+\sigma \pi_{t} \mathrm{~d} \hat{W}_{t}\right], \\
X_{0}^{\pi} & =x_{0}, \\
\mathrm{~d} p_{k}(t) & =\sum_{j} q_{j k} p_{j}(t) \mathrm{d} t+\frac{1}{\sigma}\left(\mu_{k}-\hat{\mu}_{t}\right) p_{k}(t) \mathrm{d} \hat{W}_{t}, \\
p_{k}(0) & =\mathrm{P}\left(\boldsymbol{Y}_{0}=\boldsymbol{e}_{k}\right), \quad k=1, \ldots, d .
\end{aligned}
$$

The wealth process is explicitly given by

$$
X_{t}^{\pi}=x_{0} \exp \left\{\int_{0}^{t}\left(r+\left(\hat{\mu}_{s}-r\right) \pi_{s}-\frac{1}{2} \sigma^{2} \pi_{s}^{2}\right) \mathrm{d} s+\int_{0}^{t} \sigma \pi_{s} \mathrm{~d} \hat{W}_{s}\right\} .
$$

The value functions in the reduced model are defined by

$$
\begin{aligned}
V_{\pi}(t, x, \boldsymbol{p}) & =\mathrm{E}^{t, x, \boldsymbol{p}}\left[U\left(X_{T}^{\pi}\right)\right] \quad \text { for all } \pi \in U[t, T], \\
V(t, x, \boldsymbol{p}) & =\sup _{\pi \in \mathcal{U}[t, T]} V_{\pi}(t, x, \boldsymbol{p}),
\end{aligned}
$$

where $\mathrm{E}^{t, x, \boldsymbol{p}}$ is the conditional expectation given that $X_{t}^{\pi}=x$ and $\boldsymbol{p}_{t}=\boldsymbol{p}$. The following result is often taken for granted; however, it has to be proved formally.

Theorem 1. For all $\pi \in \mathcal{U}[t, T]$ and $x>0$,

$$
V_{\pi}\left(t, x, \boldsymbol{p}_{t}\right)=\tilde{V}_{\pi}(t, x) \text { and } V\left(t, x, \boldsymbol{p}_{t}\right)=\tilde{V}(t, x) .
$$

The proof follows directly from Lemma 1 . The reduced model is now one with complete observation. We will solve it with the help of the HJB equation.

\section{Logarithmic utility}

In this section, we assume that the utility function is given by $U(x)=\log (x)$. For $\pi \in$ $\mathcal{U}[t, T]$, with the additional assumption that $\pi_{s} \in[-M, M]$ for $M \in \mathbb{R}_{+}$large, we find, from the explicit solution for $X_{t}^{\pi}$, that

$$
V_{\pi}(t, x, \boldsymbol{p})=\log (x)+h_{\pi}(t, \boldsymbol{p}),
$$

where

$$
\begin{aligned}
h_{\pi}(t, \boldsymbol{p}) & =\mathrm{E}^{t, \boldsymbol{p}}\left[\int_{t}^{T}\left(r+\left(\mu_{t}-r\right) \pi_{s}-\frac{1}{2} \sigma^{2} \pi_{s}^{2}\right) \mathrm{d} s+\int_{t}^{T} \sigma \pi_{s} \mathrm{~d} W_{s}\right] \\
& =\mathrm{E}^{t, \boldsymbol{p}}\left[\int_{t}^{T}\left(r+\left(\mu_{t}-r\right) \pi_{s}-\frac{1}{2} \sigma^{2} \pi_{s}^{2}\right) \mathrm{d} s\right]
\end{aligned}
$$

and $\mathrm{E}^{t, \boldsymbol{p}}$ is the conditional expectation given that $\boldsymbol{p}_{t}=\boldsymbol{p}$. Note that we need $\pi_{s} \in[-M, M]$ in order to have $\mathrm{E}\left[\int_{0}^{T} \pi_{s} \mathrm{~d} W_{s}\right]=0$, and that $h_{\pi}$ does not depend on $x$. 
Let $\&$ denote the probability simplex in $\mathbb{R}^{d}$. The results of the following lemma are now obvious.

Lemma 2. (a) For all $t \in[0, T], x>0$, and $p \in \&$, we have

$$
V(t, x, \boldsymbol{p})=\log (x)+h(t, \boldsymbol{p}),
$$

where $h(t, \boldsymbol{p})=\sup _{\pi \in U[t, T]} h_{\pi}(t, \boldsymbol{p})$.

(b) Let $\pi_{t}^{*}=\left(\hat{\mu}_{t}-r\right) / \sigma^{2}$ for all $t \in[0, T]$. Then, $\pi^{*}=\left(\pi_{t}^{*}\right)$ is an optimal portfolio strategy for the given investment problem.

Proof. Part (a) follows from the considerations preceding Lemma 2. Part (b) follows directly from a pathwise maximization and the fact that taking the limit $M \rightarrow \infty$ does not change the optimal investment strategy.

In the case of complete observation, i.e. where we can observe the drift process $\mu_{t}$, it is well known that the optimal investment strategy at time $t$ is to invest a fraction $\left(\mu_{t}-r\right) / \sigma^{2}$ of the wealth in the stock. Lemma 2(b) shows that the so-called certainty equivalence principle holds, i.e. the unknown appreciation rate $\mu_{t}$ is replaced by the estimate $\hat{\mu}_{t}=\mathrm{E}\left[\mu_{t} \mid \mathcal{F}_{t}^{S}\right]$ in the optimal portfolio strategy (see Kuwana (1991)).

\section{Power utility}

Suppose that the utility function is given by $U(x)=x^{\alpha} / \alpha$, with $\alpha<1$ and $\alpha \neq 0$. It is well known that, in this case, the value function can be written in the form $V(t, x, \boldsymbol{p})=$ $(1 / \alpha) x^{\alpha} g(t, \boldsymbol{p})^{1-\alpha}$. One of our main contributions in this paper is to show that the corresponding portfolio optimization problem has a smooth value function, where $g$ can be identified as a classical solution of a linear parabolic differential equation. This is not the case when the drift process $\mu_{t}$ is more general (see Zariphopoulou (2001)). In particular, we can circumvent the use of viscosity solutions. In the following, the subscripts on $g$ denote single or multiple partial differentiation with respect to the corresponding variables. Our theorem is as follows.

Theorem 2. (a) The value function $V$ of our investment problem is given by

$$
V(t, x, \boldsymbol{p})=\frac{1}{\alpha} x^{\alpha} g(t, \boldsymbol{p})^{1-\alpha} \quad \text { for all }(t, x, \boldsymbol{p}) \in[0, T] \times \mathbb{R}_{+} \times s,
$$

where $g \geq 0$ is a classical solution of the following linear parabolic differential equation, with $g(T, \boldsymbol{p})=1$ for all $\boldsymbol{p} \in$ \&:

$$
\begin{aligned}
0=g_{t} & +\frac{\alpha}{1-\alpha}\left\{r+\frac{1}{2(1-\alpha)} \frac{\left(\boldsymbol{\mu}^{\top} \boldsymbol{p}-r\right)^{2}}{\sigma^{2}}\right\} g \\
& +\sum_{k}\left\{\sum_{j} q_{j k} p_{j}+\frac{\alpha}{1-\alpha} p_{k}\left(\mu_{k}-\boldsymbol{\mu}^{\top} \boldsymbol{p}\right) \frac{\boldsymbol{\mu}^{\top} \boldsymbol{p}-r}{\sigma^{2}}\right\} g_{p_{k}} \\
& +\frac{1}{2 \sigma^{2}} \sum_{k, j}\left(\mu_{k}-\boldsymbol{\mu}^{\top} \boldsymbol{p}\right)\left(\mu_{j}-\boldsymbol{\mu}^{\top} \boldsymbol{p}\right) p_{k} p_{j} g_{p_{k} p_{j} .}
\end{aligned}
$$


(b) The optimal portfolio strategy $\pi^{*}=\left(\pi_{t}^{*}\right) \in \mathcal{U}[0, T]$ is given in feedback form as $\pi_{t}^{*}=$ $u^{*}\left(t, \boldsymbol{p}_{t}\right)$, where the function $u^{*}$ is given by

$$
u^{*}(t, \boldsymbol{p})=\frac{1}{1-\alpha} \frac{\boldsymbol{\mu}^{\top} \boldsymbol{p}-r}{\sigma^{2}}+\frac{\sum_{k} p_{k}\left(\mu_{k}-\boldsymbol{\mu}^{\top} \boldsymbol{p}\right) g_{p_{k}}(t, \boldsymbol{p})}{\sigma^{2} g(t, \boldsymbol{p})} .
$$

(c) The Feynman-Kac formula yields the following stochastic representation of $g$ :

$$
g(t, \boldsymbol{p})=\mathrm{E}\left[\exp \left(r \frac{\alpha}{1-\alpha}(T-t)+\int_{t}^{T} \frac{\alpha}{2(1-\alpha)^{2}} \frac{\left(\boldsymbol{\mu}^{\top} \boldsymbol{Z}_{s}-r\right)^{2}}{\sigma^{2}} \mathrm{~d} s\right) \mid \boldsymbol{Z}_{t}=\boldsymbol{p}\right]
$$

Here, the stochastic process $\left(\boldsymbol{Z}_{t}\right) \in \mathbb{R}^{d}$ is a (componentwise) solution of the stochastic differential equation

$$
\mathrm{d} Z_{t}^{k}=a_{k}\left(\boldsymbol{Z}_{t}\right) \mathrm{d} t+\sum_{j} b_{k, j}\left(\boldsymbol{Z}_{t}\right) \mathrm{d} W_{t}^{j}
$$

with

$$
\begin{aligned}
a_{k}(\boldsymbol{p}) & :=\sum_{j} q_{j k} p_{j}+\frac{\alpha}{1-\alpha} p_{k}\left(\mu_{k}-\boldsymbol{\mu}^{\top} \boldsymbol{p}\right) \frac{\boldsymbol{\mu}^{\top} \boldsymbol{p}-r}{\sigma^{2}}, \\
b_{k, j}(\boldsymbol{p}) & :=\frac{1}{\sigma^{2}}\left(\mu_{k}-\boldsymbol{\mu}^{\top} \boldsymbol{p}\right)\left(\mu_{j}-\boldsymbol{\mu}^{\top} \boldsymbol{p}\right) p_{k} p_{j} .
\end{aligned}
$$

Remark 1. (a) In the case in which the investor is able to observe the driving Markov chain, the optimal fraction of wealth invested in the stock at time $t$, when the Markov chain is in state $\boldsymbol{e}_{k}$, is given by

$$
u_{\mathrm{o}}^{*}\left(t, \boldsymbol{e}_{k}\right)=\frac{1}{1-\alpha} \frac{\mu_{k}-r}{\sigma^{2}}
$$

(see, e.g. Bäuerle and Rieder (2004)). Thus, in the unobservable case, the optimal fraction invested consists of the same myopic part and an additional term, which we call the drift risk. This term is sometimes also called market risk or hedging demand but, since it stems from the unknown drift rate only, we have decided to call it drift risk.

(b) Note that, since $g$ and $g_{p_{k}}$ are continuous and $[0, T] \times \&$ is compact, the optimal portfolio strategy $\left(\pi_{t}^{*}\right)$ is bounded.

The proof of Theorem 2 is given in Section 6.

\section{The HJB equation and the proof of Theorem 2}

In order to solve the investment problem, a classical approach in stochastic control theory is to examine the so-called Hamilton-Jacobi-Bellman equation. For our problem, it turns out to be

$$
\begin{aligned}
0=\sup _{u \in \mathbb{R}}\left\{v_{t}+x\left[r+u\left(\boldsymbol{\mu}^{\top} \boldsymbol{p}-r\right)\right] v_{x}+\frac{1}{2} x^{2} u^{2} \sigma^{2} v_{x x}\right. \\
+\sum_{k, j} q_{j k} p_{j} v_{p_{k}}+\sum_{k} x u p_{k}\left(\mu_{k}-\boldsymbol{\mu}^{\top} \boldsymbol{p}\right) v_{x p_{k}} \\
\left.\quad+\frac{1}{2 \sigma^{2}} \sum_{k, j}\left(\mu_{k}-\boldsymbol{\mu}^{\top} \boldsymbol{p}\right)\left(\mu_{j}-\boldsymbol{\mu}^{\top} \boldsymbol{p}\right) p_{k} p_{j} v_{p_{k} p_{j}}\right\},
\end{aligned}
$$


with the boundary condition $v(T, x, \boldsymbol{p})=x^{\alpha} / \alpha$ for all $x \in \mathbb{R}_{+}$and $\boldsymbol{p} \in \S$. The subscripts on $v$ denote partial differentiation, as for $g$ in (2). In what follows, we abbreviate the expression in curly brackets to $\mathcal{A} v(t, x, \boldsymbol{p}, u)$. To prove Theorem 2, we proceed as follows.

Theorem 3. The function $v(t, x, \boldsymbol{p}):=(1 / \alpha) x^{\alpha} g(t, \boldsymbol{p})^{1-\alpha}$, with $g$ as given in (2), is a solution of the HJB equation (3).

Proof. First, note that the boundary condition $v(T, x, \boldsymbol{p})=x^{\alpha} / \alpha$ is satisfied. Moreover, since the coefficients of the linear parabolic differential equation for $g$ are polynomials in the components of $\boldsymbol{p}$, the function $g$ is sufficiently differentiable (see, e.g. Kloeden and Platen (1995, p. 153)). We first compute the derivatives of $v$ :

$$
\begin{aligned}
v_{t} & =\frac{1}{\alpha} x^{\alpha}(1-\alpha) g^{-\alpha} g_{t}, \\
v_{x} & =x^{\alpha-1} g^{1-\alpha}, \\
v_{x x} & =(\alpha-1) x^{\alpha-2} g^{1-\alpha}, \\
v_{p_{k}} & =\frac{1}{\alpha} x^{\alpha}(1-\alpha) g^{-\alpha} g_{p_{k}}, \\
v_{x p_{k}} & =x^{\alpha-1}(1-\alpha) g^{-\alpha} g_{p_{k}}, \\
v_{p_{k} p_{j}} & =\frac{1}{\alpha} x^{\alpha}(1-\alpha)\left(g^{-\alpha} g_{p_{k} p_{j}}-\alpha g^{-\alpha-1} g_{p_{j}} g_{p_{k}}\right) .
\end{aligned}
$$

Substituting these into the HJB equation gives us

$$
\begin{aligned}
0=\sup _{u \in \mathbb{R}} \frac{1}{\alpha}\{ & g_{t}+\left[r+u\left(\boldsymbol{\mu}^{\top} \boldsymbol{p}-r\right)\right] \frac{\alpha}{1-\alpha} g-\frac{1}{2} \alpha u^{2} \sigma^{2} g \\
& +\sum_{k, j} q_{j k} p_{j} g_{p_{k}}+\sum_{k} u p_{k}\left(\mu_{k}-\boldsymbol{\mu}^{\top} \boldsymbol{p}\right) \alpha g_{p_{k}} \\
& \left.+\frac{1}{2 \sigma^{2}} \sum_{k, j}\left(\mu_{k}-\boldsymbol{\mu}^{\top} \boldsymbol{p}\right)\left(\mu_{j}-\boldsymbol{\mu}^{\top} \boldsymbol{p}\right) p_{k} p_{j}\left(g_{p_{k} p_{j}}-\alpha g^{-1} g_{p_{j}} g_{p_{k}}\right)\right\}
\end{aligned}
$$

after some simple algebra. (Note that we need $\alpha<1$ here.) Since $g \geq 0$, the maximum point is well defined and given by

$$
\frac{1}{1-\alpha} \frac{\boldsymbol{\mu}^{\top} \boldsymbol{p}-r}{\sigma^{2}}+\frac{\sum_{k} p_{k}\left(\mu_{k}-\boldsymbol{\mu}^{\top} \boldsymbol{p}\right) g_{p_{k}}}{\sigma^{2} g} .
$$

Inserting the maximum point and simplifying the expression, we find that $g$ has to satisfy the partial differential equation (2), in agreement with the statement of the theorem.

The power change of variable for the value function has already been used by Zariphopoulou (2001) and Pham (2002). Here, it is shown that this trick also works in a multidimensional setting. The next theorem provides the verification that $v(t, x, \boldsymbol{p})$ as given in Theorem 3 is indeed the value function of our investment problem.

Theorem 4. Suppose that $v(t, x, \boldsymbol{p})$ is as given in Theorem 3. Then,

(a) $V(t, x, \boldsymbol{p})=v(t, x, \boldsymbol{p})$ for all $(t, x, \boldsymbol{p}) \in[0, T] \times \mathbb{R}_{+} \times \delta$; and

(b) the optimal portfolio strategy $\pi^{*}=\left(\pi_{t}^{*}\right) \in U[0, T]$ is as given in Theorem $2(b)$. 
Proof. We explicitly consider the case $\alpha \in(0,1)$; the case $\alpha<0$ can be shown similarly. Let $\pi \in U[t, T]$ be an arbitrary portfolio strategy and $\left(X_{t}^{\pi}\right)$ the corresponding wealth process. We interpret $v(t, x, \boldsymbol{p})$ as a function on $\mathbb{R}_{+} \times \mathbb{R}^{d+1}$. Since $v$ is sufficiently smooth, we can apply Itô's formula to obtain

$$
\begin{aligned}
v\left(T, X_{T}^{\pi}, \boldsymbol{p}_{T}\right)= & v(t, x, \boldsymbol{p})+\int_{t}^{T} \mathcal{A} v\left(s, X_{s}^{\pi}, \boldsymbol{p}_{s}, \pi_{s}\right) \mathrm{d} s \\
& +\int_{t}^{T} v_{x}\left(s, X_{s}^{\pi}, \boldsymbol{p}_{s}\right) X_{s}^{\pi} \pi_{s} \sigma \mathrm{d} \hat{W}_{s} \\
& +\int_{t}^{T} \sum_{k} v_{p_{k}}\left(s, X_{s}^{\pi}, \boldsymbol{p}_{s}\right) \frac{1}{\sigma} p_{k}(s)\left(\mu_{k}-\boldsymbol{\mu}^{\top} \boldsymbol{p}_{s}\right) \mathrm{d} \hat{W}_{s} \\
\leq & v(t, x, \boldsymbol{p})+\int_{t}^{T}\left(X_{s}^{\pi}\right)^{\alpha} g\left(s, \boldsymbol{p}_{s}\right)^{1-\alpha} \pi_{s} \sigma \mathrm{d} \hat{W}_{s} \\
& +\int_{t}^{T} \sum_{k}\left(X_{s}^{\pi}\right)^{\alpha} \frac{g_{p_{k}}\left(s, \boldsymbol{p}_{s}\right)}{g\left(s, \boldsymbol{p}_{s}\right)^{\alpha}} \frac{1-\alpha}{\alpha \sigma} p_{k}(s)\left(\mu_{k}-\boldsymbol{\mu}^{\top} \boldsymbol{p}_{s}\right) \mathrm{d} \hat{W}_{s},
\end{aligned}
$$

where the inequality follows from the HJB equation. Since $v \geq 0$, the local martingale $\left(\int_{t}^{T} \cdots \mathrm{d} \hat{W}_{s}\right)_{T \geq t}$ is bounded from below by $-v(t, x, \boldsymbol{p})$ and, thus, is a supermartingale. Taking the conditional expectation and using the boundary condition for $v$, we find that

$$
\mathrm{E}^{t, x, p}\left[\frac{1}{\alpha}\left(X_{T}^{\pi}\right)^{\alpha}\right] \leq v(t, x, \boldsymbol{p}) .
$$

Since $\pi$ was arbitrary, we obtain $V(t, x, \boldsymbol{p}) \leq v(t, x, \boldsymbol{p})$. Now suppose that $\left(\pi_{t}^{*}\right)$ is as in part (b). Since $\left(\pi_{t}^{*}\right)$ maximizes the HJB equation, we obtain equality in (4) under $\left(\pi_{t}^{*}\right)$. Note that, since $\left(\pi_{t}^{*}\right)$ is bounded, $g$ and $g_{p_{k}}$ are continuous, and $[0, T] \times \delta$ is compact, the local martingales $\int \cdots \mathrm{d} \hat{W}_{s}$ are true martingales. Taking the expectation, we now obtain

$$
\mathrm{E}^{t, x, p}\left[\frac{1}{\alpha}\left(X_{T}^{\pi}\right)^{\alpha}\right]=v(t, x, \boldsymbol{p}),
$$

and the statement follows.

Thus, parts (a) and (b) of Theorem 2 are proven. The Feynman-Kac formula (Theorem 2(c)) is standard; see, e.g. Kloeden and Platen (1995, p. 153).

\section{The Bayesian case}

In this section, we consider a special case of the previously discussed model, namely the so-called Bayesian case. Here, the unobserved drift process $\left(\mu_{t}\right)$ is simply a random variable $\mu_{t}=\theta$ that does not change over time, and the investor knows the initial distribution $\mathrm{P}\left(\theta=\mu_{k}\right)=: p_{k}, k=1, \ldots, d$. The possible values $\theta$ can take are $\mu_{1}, \ldots, \mu_{d}$. As before, we assume that $\theta$ and $\left(W_{t}\right)$ are independent. This model has already been solved by Karatzas and Zhao (2001) via the martingale method and by stochastic control. We now relate their result to our model of Section 2, giving a self-contained proof. Formally, we obtain the Bayesian case if we set the intensity matrix $\boldsymbol{Q} \equiv \mathbf{0}$ in the model of Section 2 . With this modification, the results of Sections 3-5 hold for the Bayesian case. However, we will see that the analysis can be simplified considerably in this setup. This is mainly due to the fact that, instead of looking 
at the $\mathbb{R}^{d+1}$-valued state process $\left(X_{t}, p_{1}(t), \ldots, p_{d}(t)\right)$ in the reduced model, we can find a sufficient statistic for the unobserved parameter $\theta$ and can restrict to a two-dimensional state space. A crucial step for this procedure is to look at the optimization problem under a change of measure. Since the logarithmic utility is quite simple, we here restrict to the power utility $U(x)=x^{\alpha} / \alpha$, with $\alpha<1$ and $\alpha \neq 0$. In the sequel, we will use the following results. Recall, from Section 2, that $\hat{\mu}_{t}=\sum_{k=1}^{d} \mu_{k} p_{k}(t)$, with $p_{k}(t)=\mathrm{P}\left(\theta=\mu_{k} \mid \mathcal{F}_{t}^{S}\right), k=1, \ldots, d$, and $\hat{W}_{t}=W_{t}+(1 / \sigma) \int_{0}^{t}\left(\theta-\hat{\mu}_{s}\right) \mathrm{d} s$. Let us now introduce the process

$$
Y_{t}:=\hat{W}_{t}+\frac{1}{\sigma} \int_{0}^{t}\left(\hat{\mu}_{s}-r\right) \mathrm{d} s=W_{t}+\frac{\theta-r}{\sigma} t .
$$

It is convenient to write $\gamma_{k}:=\left(\mu_{k}-r\right) / \sigma$ and

$$
L_{t}\left(\mu_{k}, y\right):= \begin{cases}\exp \left(\gamma_{k} y-\frac{1}{2} \gamma_{k}^{2} t\right), & t>0, \\ 1, & t=0,\end{cases}
$$

for $t \in[0, T], y \in \mathbb{R}$, and $k=1, \ldots, d$. It is well known that $\left(L_{t}^{-1}\left(\theta, Y_{t}\right)\right)$ is a martingale density process with respect to the filtration $\mathfrak{F}^{\theta, W}$ generated by $\theta$ and $\left(W_{t}\right)$. Then we can define a new probability measure $\mathrm{Q}$ by $\mathrm{dQ} / \mathrm{dP}=L_{T}^{-1}\left(\theta, Y_{T}\right)$. Under $\mathrm{Q}$, the process $\left(W_{t}^{\mathrm{Q}}\right)$, with $W_{t}^{\mathrm{Q}}:=Y_{t}$, is a Brownian motion with respect to $\mathfrak{F}^{\theta, W}$. The process $\left(L_{t}\left(\theta, Y_{t}\right)\right)$ is a Q-martingale with respect to $\mathfrak{F}^{\theta, W}$. Note that it can be shown that $\theta$ and $\left(W_{t}^{\mathrm{Q}}\right)$ are independent under Q. Finally, for $t \in[0, T]$ and $y \in \mathbb{R}$, we use the abbreviation

$$
F(t, y):=\sum_{k=1}^{d} L_{t}\left(\mu_{k}, y\right) p_{k} .
$$

Now we are able to state our first result.

Lemma 3. With the notation introduced in this section,

(a) $\mathfrak{F}^{S}=\mathfrak{F}^{Y}$, i.e. the filtration generated by $\left(S_{t}\right)$ is the same as the one generated by $\left(Y_{t}\right)=\left(W_{t}^{\mathrm{Q}}\right) ;$ and

(b) $p_{k}(t)$ depends only on $Y_{t}$. More precisely, for $k=1, \ldots, d$,

$$
p_{k}(t)=\mathrm{P}\left(\theta=\mu_{k} \mid \mathcal{F}_{t}^{S}\right)=\frac{L_{t}\left(\mu_{k}, Y_{t}\right) p_{k}}{F\left(t, Y_{t}\right)} .
$$

Proof. Part (a) follows from the definitions of the stock price process $\left(S_{t}\right)$ and $\left(\hat{W}_{t}\right)$. The Bayes formula for conditional expectations reads

$$
\mathrm{E}\left[Z \mid \mathcal{F}_{t}^{S}\right]=\frac{\mathrm{E}_{\mathrm{Q}}\left[Z L_{T}\left(\theta, Y_{T}\right) \mid \mathcal{F}_{t}^{S}\right]}{\mathrm{E}_{\mathrm{Q}}\left[L_{T}\left(\theta, Y_{T}\right) \mid \mathcal{F}_{t}^{S}\right]},
$$

where $Z$ is a random variable defined on our probability space. Substituting $Z=\mathbf{1}_{\left\{\theta=\mu_{k}\right\}}$ in this yields the result of part (b).

In particular, Lemma 3 implies that $\hat{\mu}_{t}=\sum_{k=1}^{d} \mu_{k} p_{k}(t)$ is a function of $Y_{t}$ alone. Therefore, $\left(Y_{t}\right)$ can be seen as a sufficient statistic. There is no need to consider the conditional probabilities $p_{k}(t)$ for all $k=1, \ldots, d$. More precisely, when we define

$$
\mu(t, y)=\frac{\sum_{k=1}^{d} \mu_{k} L_{t}\left(\mu_{k}, y\right) p_{k}}{\sum_{k=1}^{d} L_{t}\left(\mu_{k}, y\right) p_{k}},
$$


we have $\hat{\mu}_{t}=\mu\left(t, Y_{t}\right)$. It is now convenient to introduce the process $\gamma_{t}=\gamma\left(t, Y_{t}\right)$, where $\gamma(t, y):=(\mu(t, y)-r) / \sigma$. We can reduce our portfolio problem to a problem with complete observation and two-dimensional state space in the following way:

$$
\begin{aligned}
\mathrm{d} X_{t}^{\pi} & =X_{t}^{\pi}\left[\left(r+\sigma \gamma_{t} \pi_{t}\right) \mathrm{d} t+\sigma \pi_{t} \mathrm{~d} \hat{W}_{t}\right], & & X_{0}^{\pi}=x_{0}, \\
\mathrm{~d} Y_{t} & =\gamma_{t} \mathrm{~d} t+\mathrm{d} \hat{W}_{t}, & & Y_{0}=0 .
\end{aligned}
$$

As in Section 3, we define the value functions $V_{\pi}(t, x, y)$ and $V(t, x, y)$ for $(t, x, y) \in[0, T] \times$ $\mathbb{R}_{+} \times \mathbb{R}$, and obtain

$$
\begin{aligned}
V_{\pi}\left(t, x, Y_{t}\right) & =\tilde{V}_{\pi}(t, x), \\
V\left(t, x, Y_{t}\right) & =\tilde{V}(t, x),
\end{aligned}
$$

as in Theorem 1 .

A proof of parts (a) and (b) of the following theorem can also be found in Theorem 3.2 of Karatzas and Zhao (2001).

Theorem 5. (a) The value function $V$ of our investment problem with power utility is given by

$$
V(t, x, y)=\frac{1}{\alpha} x^{\alpha} g(t, y)^{1-\alpha}
$$

for all $(t, x, y) \in[0, T] \times \mathbb{R}_{+} \times \mathbb{R}$, where $g(t, y) \geq 0$ is a classical solution of the linear parabolic differential equation

$$
0=g_{t}+\frac{\alpha}{1-\alpha}\left\{r+\frac{1}{2} \frac{\gamma(t, y)^{2}}{1-\alpha}\right\} g+\frac{\gamma(t, y)}{1-\alpha} g_{y}+\frac{1}{2} g_{y y},
$$

with $g(T, y)=1$ for all $y \in \mathbb{R}$.

(b) The following representation of $g$ holds for $t \in[0, T]$ and $y \in \mathbb{R}$ :

$$
g(t, y)=\mathrm{E}\left[\left(\frac{F\left(T, Y_{T}\right) B_{T}}{F\left(t, Y_{t}\right) B_{t}}\right)^{\alpha /(1-\alpha)} \mid Y_{t}=y\right] .
$$

(c) The optimal portfolio strategy $\pi^{*}=\left(\pi_{t}^{*}\right) \in U[0, T]$ is given in feedback form as $\pi_{t}^{*}=$ $u^{*}\left(t, Y_{t}\right)$, where the function $u^{*}$ is given by

$$
u^{*}(t, y)=\frac{1}{1-\alpha} \frac{\mu(t, y)-r}{\sigma^{2}}+\frac{g_{y}(t, y)}{\sigma g(t, y)} .
$$

Proof. It is straightforward to see that our portfolio problem is equivalent to the following optimization problem (with respect to the equivalent martingale measure Q), where we maximize over all $\pi \in \mathcal{U}[0, T]$ :

$$
\begin{aligned}
& \mathrm{E}_{\mathrm{Q}}\left[F\left(T, Y_{T}\right) \frac{1}{\alpha}\left(X_{T}^{\pi}\right)^{\alpha}\right] & \rightarrow \max , \\
\mathrm{d} X_{t}^{\pi}=X_{t}^{\pi}\left(r \mathrm{~d} t+\sigma \pi_{t} \mathrm{~d} W_{t}^{\mathrm{Q}}\right), & & X_{0}^{\pi}=x_{0}, \\
\mathrm{~d} Y_{t}=\mathrm{d} W_{t}^{\mathrm{Q}}, & & Y_{0}=0 .
\end{aligned}
$$

We denote the value function of this problem by $V^{\mathrm{Q}}(t, x, y)$. It follows from the definition that $V\left(0, x_{0}, 0\right)=V^{\mathrm{Q}}\left(0, x_{0}, 0\right)$. 
(a) Solving the HJB equation for the Q-problem, we obtain

$$
V^{\mathrm{Q}}(t, x, y)=\frac{1}{\alpha} x^{\alpha} g^{\mathrm{Q}}(t, y)^{1-\alpha},
$$

as in Section 6, where $g^{\mathrm{Q}}$ is a classical solution of the linear parabolic differential equation

$$
0=g_{t}^{\mathrm{Q}}+\frac{\alpha r}{1-\alpha} g^{\mathrm{Q}}+\frac{1}{2} g_{y y}^{\mathrm{Q}},
$$

with $g^{\mathrm{Q}}(T, y)=F(T, y)^{1 /(1-\alpha)}$ for all $y \in \mathbb{R}$. Moreover, the Feynman-Kac formula gives the representation

$$
g^{\mathrm{Q}}(t, y)=\exp \left(r(T-t) \frac{\alpha}{1-\alpha}\right) \mathrm{E}_{\mathrm{Q}}\left[F\left(T, Y_{T}\right)^{1 /(1-\alpha)} \mid Y_{t}=y\right] .
$$

If we define $g(t, y):=F(t, y)^{1 /(\alpha-1)} g^{\mathrm{Q}}(t, y)$ then it is easy to see, after some calculations, that $g$ is a classical solution to the HJB equation for the P-problem equivalent to (5).

(b) Using the representation of $g^{\mathrm{Q}}$ just given, and applying the Bayes formula, yields the statement for $g(t, y)$.

(c) From the HJB equation, we find that the optimal portfolio strategy is given by $\pi_{t}^{*}=u^{*}\left(t, Y_{t}\right)$, where the function $u^{*}$ is given as stated.

Remark 2. Note that the optimal portfolio strategy $\left(\pi_{t}^{*}\right)$, with $\pi_{t}^{*}=u^{*}\left(t, Y_{t}\right)$, can also be written as

$$
u^{*}(t, y)=\frac{g_{y}^{\mathrm{Q}}(t, y)}{\sigma g^{\mathrm{Q}}(t, y)} .
$$

This follows from the equivalent Q-problem formulated in the proof of Theorem 5.

\section{Properties of the optimal investment strategy in the Bayesian case}

In this section, we investigate the structural properties of the optimal investment fraction $u^{*}(t, y)$ given in Theorem 5(c). In particular, we will compare the optimal investment strategy with the one we obtain when the drift rate is known. In the observable case, the problem has been solved by Merton (1971), (1973). Suppose that

$$
\mathrm{d} S_{t}=S_{t}\left(\mu \mathrm{d} t+\sigma \mathrm{d} W_{t}\right)
$$

describes the dynamics of the stock price and that $\mu \in \mathbb{R}$ is observable. Then, it is well known that it is optimal to invest the constant fraction

$$
\frac{1}{1-\alpha} \frac{\mu-r}{\sigma^{2}}
$$

of the wealth in the stock. Let us now assume that we are in the Bayesian case and observe that the state of $Y_{t}$ is $y$ at time $t$. Then we expect that the drift rate of the stock is $\mu(t, y)$. If we were certain that the drift rate were $\mu(t, y)$ then we would invest the fraction

$$
u_{\mathrm{o}}^{*}(t, y, \alpha)=\frac{1}{1-\alpha} \frac{\mu(t, y)-r}{\sigma^{2}}=\frac{1}{(1-\alpha) \sigma} \gamma(t, y)
$$

of the wealth in the stock. Recall that $u^{*}(t, y)=g_{y}^{\mathrm{Q}}(t, y) / \sigma g^{\mathrm{Q}}(t, y)$ and $u^{*}$ depends on $\alpha$. Hence, we will write $u^{*}(t, y) \equiv u^{*}(t, y, \alpha)$. We now obtain the following comparisons, where part (d) is nontrivial and of particular interest in practical applications. 
Theorem 6. (a) At time $T$ the optimal fraction is equal to the myopic part, i.e.

$$
\lim _{t \rightarrow T} u^{*}(t, y, \alpha)=u_{\mathrm{o}}^{*}(T, y, \alpha)
$$

for all $y \in \mathbb{R}$ and $\alpha<1$.

(b) As $\alpha \rightarrow 0$, the optimal fraction tends to the myopic part, i.e.

$$
\lim _{\alpha \rightarrow 0} u^{*}(t, y, \alpha)=u_{\mathrm{o}}^{*}(t, y, 0)
$$

for all $y \in \mathbb{R}$ and $t \in[0, T]$.

(c) Suppose that $\mu_{1} \leq \cdots \leq \mu_{d}$. Then we obtain the following bounds for all $y \in \mathbb{R}$ and $t \in[0, T]$ :

$$
\frac{1}{1-\alpha} \frac{\mu_{1}-r}{\sigma^{2}} \leq u^{*}(t, y, \alpha) \leq \frac{1}{1-\alpha} \frac{\mu_{d}-r}{\sigma^{2}} .
$$

(d) Suppose that $r \leq \mu_{1} \leq \cdots \leq \mu_{d}$. If $\alpha \in(0,1)$ then

$$
u^{*}(t, y, \alpha) \geq u_{\mathrm{o}}^{*}(t, y, \alpha)
$$

and, if $\alpha<0$, then

$$
u^{*}(t, y, \alpha) \leq u_{\mathrm{o}}^{*}(t, y, \alpha)
$$

for all $y \in \mathbb{R}$ and $t \in[0, T]$.

Proof. Let us define $\gamma^{*}(t, y, \alpha):=(1-\alpha) g_{y}^{\mathrm{Q}}(t, y) / g^{\mathrm{Q}}(t, y)$, as it is then sufficient to prove the statements for $\gamma^{*}(t, y, \alpha)$ and $\gamma(t, y)$. From Section 7, we know that $\gamma^{*}(t, y, \alpha)=$ $\Gamma_{\mathrm{D}}(t, y, \alpha) / \Gamma_{\mathrm{N}}(t, y, \alpha)$ with

$$
\begin{aligned}
\Gamma_{\mathrm{N}}(t, y, \alpha) & :=\int_{\mathbb{R}} F(T, y+x)^{1 /(1-\alpha)} \phi_{T-t}(x) \mathrm{d} x, \\
\Gamma_{\mathrm{D}}(t, y, \alpha) & :=\int_{\mathbb{R}} F(T, y+x)^{\alpha /(1-\alpha)}\left(\sum_{k=1}^{d} p_{k} \gamma_{k} L_{T}\left(\mu_{k}, y+x\right)\right) \phi_{T-t}(x) \mathrm{d} x,
\end{aligned}
$$

where $\phi_{T-t}$ is the density of the normal distribution with expectation 0 and variance $T-t$.

(a) In this case, the following representations of $\Gamma_{\mathrm{D}}(t, y, \alpha)$ and $\Gamma_{\mathrm{N}}(t, y, \alpha)$ are useful:

$$
\begin{aligned}
& \Gamma_{\mathrm{N}}(t, y, \alpha)=\mathrm{E}\left[F\left(T, y+W_{T-t}\right)^{1 /(1-\alpha)}\right], \\
& \Gamma_{\mathrm{D}}(t, y, \alpha)=\mathrm{E}\left[F\left(T, y+W_{T-t}\right)^{\alpha /(1-\alpha)}\left(\sum_{k=1}^{d} p_{k} \gamma_{k} L_{T}\left(\mu_{k}, y+W_{T-t}\right)\right)\right] .
\end{aligned}
$$

Since $\lim _{t \rightarrow T} W_{T-t}=0$ almost surely, we obtain

$$
\begin{aligned}
& \lim _{t \rightarrow T} \Gamma_{\mathrm{N}}(t, y, \alpha)=F(T, y)^{1 /(1-\alpha)}, \\
& \lim _{t \rightarrow T} \Gamma_{\mathrm{D}}(t, y, \alpha)=F(T, y)^{\alpha /(1-\alpha)}\left(\sum_{k=1}^{d} p_{k} \gamma_{k} L_{T}\left(\mu_{k}, y\right)\right),
\end{aligned}
$$


due to the continuity of the functions involved. Altogether, this yields

$$
\lim _{t \rightarrow T} \gamma^{*}(t, y, \alpha)=\frac{\sum_{k=1}^{d} p_{k} \gamma_{k} L_{T}\left(\mu_{k}, y\right)}{\sum_{k=1}^{d} p_{k} L_{T}\left(\mu_{k}, y\right)}=\gamma(T, y) .
$$

(b) We obtain

$$
\begin{aligned}
& \lim _{\alpha \rightarrow 0} \Gamma_{\mathrm{N}}(t, y, \alpha)=\sum_{k=1}^{d} p_{k} \int_{\mathbb{R}} L_{T}\left(\mu_{k}, y+x\right) \phi_{T-t}(x) \mathrm{d} x, \\
& \lim _{\alpha \rightarrow 0} \Gamma_{\mathrm{D}}(t, y, \alpha)=\sum_{k=1}^{d} p_{k} \gamma_{k} \int_{\mathbb{R}} L_{T}\left(\mu_{k}, y+x\right) \phi_{T-t}(x) \mathrm{d} x .
\end{aligned}
$$

Moreover,

$$
\int_{\mathbb{R}} L_{T}\left(\mu_{k}, y+x\right) \phi_{T-t}(x) \mathrm{d} x=L_{t}\left(\mu_{k}, y\right),
$$

which implies the result.

(c) Since $L_{T}, p_{k} \geq 0$, we obtain

$$
\gamma_{1} F(T, y+x) \leq \sum_{k=1}^{d} p_{k} \gamma_{k} L_{T}\left(\mu_{k}, y+x\right) \leq \gamma_{d} F(T, y+x) .
$$

Hence, we can bound the denominator of $\gamma^{*}(t, y, \alpha)$ by

$$
\gamma_{1} \Gamma_{\mathrm{N}}(t, y, \alpha) \leq \Gamma_{\mathrm{D}}(t, y, \alpha) \leq \gamma_{d} \Gamma_{\mathrm{N}}(t, y, \alpha)
$$

and the result follows.

(d) Suppose that $\alpha \in(0,1)$. We have to show that $\gamma(t, y) \leq \gamma^{*}(t, y, \alpha)$. Both sides can be interpreted as expectations in the following way:

$$
\gamma(t, y)=\sum_{k=1}^{d} \gamma_{k} p_{k}(t, y)
$$

where

$$
p_{k}(t, y)=\frac{p_{k} L_{t}\left(\mu_{k}, y\right)}{F(t, y)}
$$

and

$$
\gamma^{*}(t, y, \alpha)=\sum_{k=1}^{d} \gamma_{k} q_{k}(t, y, \alpha)
$$

where

$$
q_{k}(t, y, \alpha)=\frac{\int_{\mathbb{R}} p_{k} L_{T}\left(\mu_{k}, y+x\right) F(T, y+x)^{\alpha /(1-\alpha)} \phi_{T-t}(x) \mathrm{d} x}{\Gamma_{\mathrm{N}}(t, y, \alpha)} .
$$

We will show now that the densities satisfy

$$
\left(p_{k}(t, y), k=1, \ldots, d\right) \leq \operatorname{lr}\left(q_{k}(t, y, \alpha), k=1, \ldots, d\right),
$$


where ' $\leq_{\mathrm{lr}}$ ' is the likelihood-ratio order, i.e. we show that

$$
\frac{q_{k}(t, y, \alpha)}{p_{k}(t, y)}
$$

is increasing in $k$ for all $t \in[0, T]$ and $y \in \mathbb{R}$. Then it is well known that the expectations are ordered as stated. Obviously,

$$
\frac{q_{k}(t, y, \alpha)}{p_{k}(t, y)}=C \frac{\int_{\mathbb{R}} L_{T}\left(\mu_{k}, y+x\right) F(T, y+x)^{\alpha /(1-\alpha)} \phi_{T-t}(x) \mathrm{d} x}{L_{t}\left(\mu_{k}, y\right)},
$$

where $C>0$ is a constant. Since $L_{T}\left(\mu_{k}, y+x\right)=L_{t}\left(\mu_{k}, y\right) L_{T-t}\left(\mu_{k}, x\right)$, it follows that

$$
\frac{q_{k}(t, y, \alpha)}{p_{k}(t, y)}=C \mathrm{E}\left[F\left(T, y+X_{k}\right)^{\alpha /(1-\alpha)}\right],
$$

where $X_{k} \sim \mathcal{N}\left(\gamma_{k}(T-t), T-t\right)$. Thus, we have to show that

$$
\mathrm{E}\left[F\left(T, y+X_{k}\right)^{\alpha /(1-\alpha)}\right] \leq \mathrm{E}\left[F\left(T, y+X_{k+1}\right)^{\alpha /(1-\alpha)}\right] .
$$

This inequality is of the form

$$
\mathrm{E}\left[f\left(X_{k}\right)\right] \leq \mathrm{E}\left[f\left(X_{k+1}\right)\right]
$$

where the function $f(x)$ is increasing in $x$ since $\alpha \in(0,1)$ and $\gamma_{k} \geq 0$. Thus, the statement is true since $X_{k} \leq_{\text {st }} X_{k+1}$, where ' $\leq_{\text {st }}$ ' is the usual stochastic order. If $\alpha \in(-\infty, 0)$ then $f(x)$ is decreasing and we obtain the reverse inequality.

Remark 3. (a) The optimal fraction $u_{\mathrm{o}}^{*}(t, y, 0)$ of Theorem 6(b) is the optimal fraction we obtain in the case of a logarithmic utility function (see Section 4). Thus, the portfolio problem with logarithmic utility can be seen as the limiting problem when $\alpha \rightarrow 0$ in the power utility case.

(b) Part (d) of Theorem 6 tells us that we have to invest more in the stock in the case of an unobservable drift rate, relative to the case in which we know that $\mu(t, y)$ is the drift rate when $\alpha \in(0,1)$. If $\alpha<0$, this conclusion is reversed. A heuristic explanation of this phenomenon is as follows. Although in all cases our investor is risk averse, the degree of risk aversion changes with $\alpha$. Formally, the degree of risk aversion is defined by the Arrow-Pratt absolute risk aversion coefficient, which is

$$
-\frac{U^{\prime \prime}(x)}{U^{\prime}(x)}=(1-\alpha) \frac{1}{x}
$$

in the case of the power utility $U(x)=x^{\alpha} / \alpha$. Thus, the risk aversion decreases for all wealth levels with $\alpha$. In particular, if $\alpha \in(0,1)$ the investor is less risk averse than in the logarithmic utility case $(\alpha=0)$ and, thus, invests more in the stock.

(c) Theorem 6(c) implies that $\lim _{\alpha \rightarrow-\infty} u^{*}(t, y, \alpha)=0$ (also see the preceding remark).

In the following figures, we have computed the optimal fractions $u^{*}(t, y, \alpha)$ and $u_{\mathrm{o}}^{*}(t, y, \alpha)$ in the case of partial and complete observation for the following data: $d=3, r=0.04, \sigma=0.2$, $\mu_{2}=0.1, \mu_{3}=0.2, t=0, y=0, p_{1}=0.2, p_{2}=0.4$, and $p_{3}=0.4$. Figure 1 shows the 


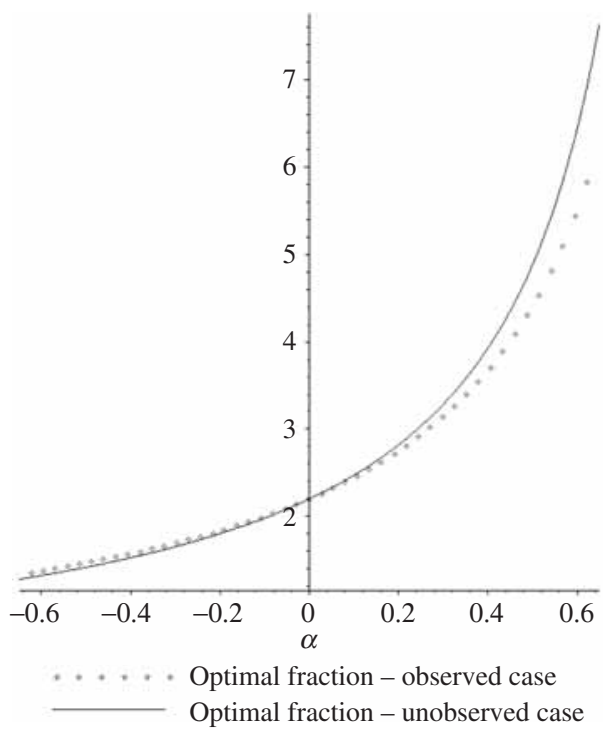

FiguRE 1.

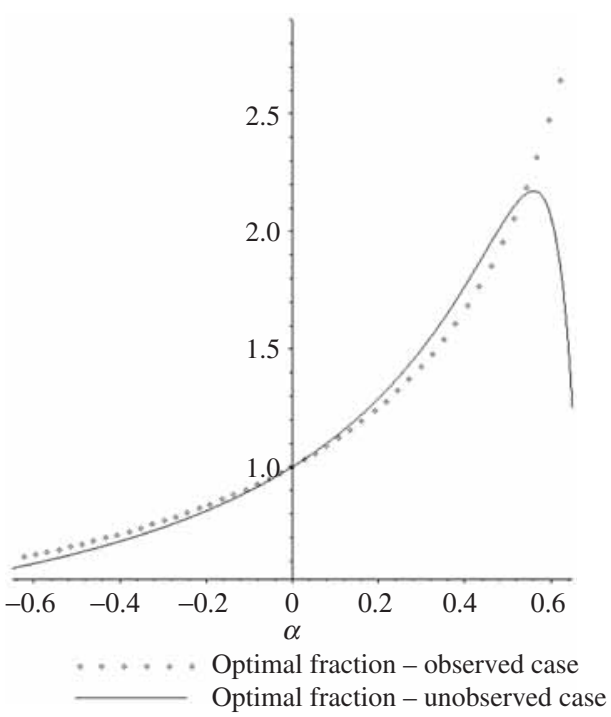

FIGURE 2.

optimal fractions that have to be invested in the stock in the observed case $u_{\mathrm{o}}^{*}(t, y, \alpha)$ and in the unobserved case $u^{*}(t, y, \alpha)$, as functions of $\alpha$, when $\mu_{1}=0.04$ and $T=1$. According to Theorem 6(b), for $\alpha=0$ the fractions coincide. Our conjecture is that both $u^{*}(t, y, \alpha)$ and the difference $u^{*}(t, y, \alpha)-u_{\mathrm{o}}^{*}(t, y, \alpha)$ are increasing in $\alpha$ if $\mu_{k} \geq r$ for all $k$. Figure 2 shows the same situation with $\mu_{1}=-0.2$, i.e. the first stock has a negative appreciation rate. In this case, we can see that Theorem 6(d) no longer holds. 


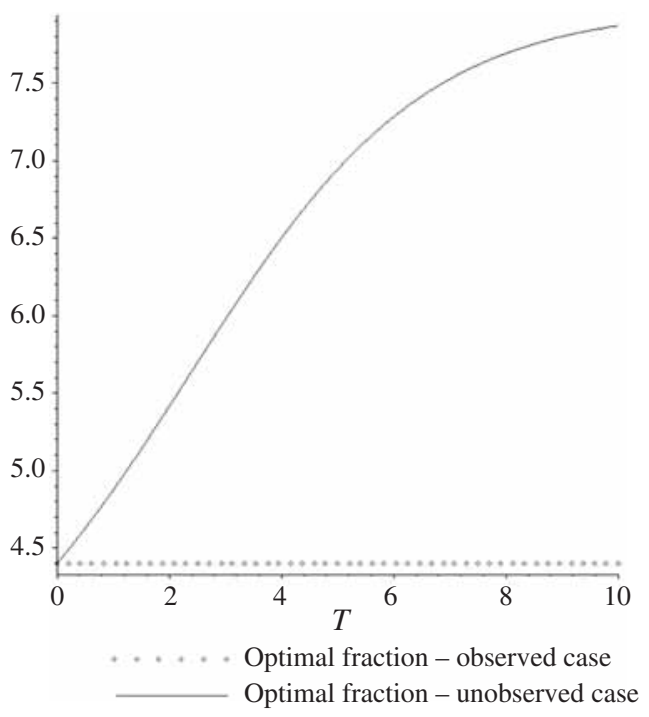

Figure 3.

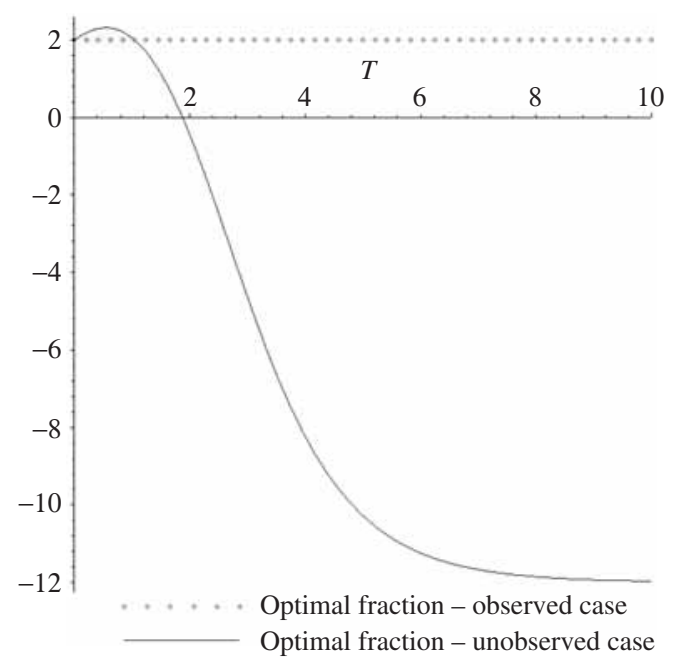

Figure 4.

Figures 3 and 4 show the optimal fractions as functions of the planning horizon $T$, with $\alpha=0.5$. As shown in Theorem 6(a), the fractions coincide for $T=t=0$. Figure 3 was computed with $\mu_{1}=0.04$ and Figure 4 with $\mu_{1}=-0.2$. In the case in which $\alpha>0$ and $\mu_{k} \geq r$ for all $k$, we conjecture that $u^{*}(t, y, \alpha)$ is increasing in $T$ and converges to the upper bound $[1 /(1-\alpha)]\left(\mu_{d}-r\right) / \sigma^{2}$. Figure 4 shows that there is no monotonicity with respect to $T$, in general. 


\section{References}

BäUerle, N. ANd Rieder, U. (2004). Portfolio optimization with Markov-modulated stock prices and interest rates. IEEE Trans. Automatic Control 49, 442-447.

Elliott, R. J., Aggoun, L. And Moore, J. B. (1994). Hidden Markov Models: Estimation and Control. Springer, New York.

Haussmann, U. G. And Sass, J. (2004). Optimal terminal wealth under partial information for HMM stock returns. In Mathematics of Finance (Contemp. Math. 351), AMS, Providence, RI, pp. 171-185.

KaratZas, I. AND ZHAO, X. (2001). Bayesian adaptive portfolio optimization. In Option Pricing, Interest Rates and Risk Management, Cambridge University Press, pp. 632-669.

Kloeden, P. E. and Platen, E. (1995). Numerical Solution of Stochastic Differential Equations. Springer, Berlin.

KuwanA, Y. (1991). Certainty equivalence and logarithmic utilities in consumption/investment problems. Math. Finance 5, 297-310.

LaKner, P. (1995). Utility maximization with partial information. Stoch. Process. Appl. 56, 247-273.

LaKner, P. (1998). Optimal trading strategy for an investor: the case of partial information. Stoch. Process. Appl. 76, 77-97.

Merton, R. C. (1971). Optimum consumption and portfolio rules in a continuous-time model. J. Econom. Theory 3, 373-413. (Correction: 6 (1973), 213-214.)

Pнам, H. (2002). Smooth solutions to optimal investment models with stochastic volatilities and portfolio constraints. Appl. Math. Optimization 46, 55-78.

RisheL, R. (1999). Optimal portfolio management with partial observation and power utility function. In Stochastic Analysis, Control, Optimization and Applications, eds W. McEneaney, G. Yin and Q. Zhang, Birkhäuser, Boston, MA, pp. 605-620.

Sass, J. And Haussmann, U. G. (2004). Optimizing the terminal wealth under partial information: the drift process as a continuous time Markov chain. Finance Stoch. 8, 553-577.

ZARIPHOPOULOU, T. (2001). A solution approach to valuation with unhedgeable risks. Finance Stoch. 5, 61-82. 